\title{
Antiphase domains in plastically deformed Zn-Mg-Dy single quasicrystals
}

\author{
M. Heggen, M. Feuerbacher, P. Schall, and K. Urban \\ Institut für Festkörperforschung, Forschungszentrum Jülich GmbH, D-52425 Jülich, Germany \\ R. Wang \\ Department of Physics, Wuhan University, 430072 Wuhan, People's Republic of China
}

(Received 17 January 2001; published 5 June 2001)

\begin{abstract}
The results of microstructural investigations of plastically deformed and undeformed $\mathrm{Zn}-\mathrm{Mg}-\mathrm{Dy}$ single quasicrystals by means of transmission electron microscopy are presented. Planar defects were observed in heat-treated quenched samples and in deformed samples, which could be identified as antiphase boundaries of the face-centered icosahedral structure. A strong increase of the density of antiphase boundaries during plastic deformation was observed, which could be attributed to the movement of partial dislocations. The plane normals of the antiphase boundaries were determined for differently treated samples. In heat-treated and quenched samples $90 \%$ of the plane normals were found to be parallel to fivefold directions. In deformed samples, planes perpendicular to pseudo-twofold $\langle 1 / 12 / 11 / 2\rangle$ directions are most frequently observed. These planes are inclined by an angle of $46^{\circ}$ with respect to the $[0 / 00 / 00 / 2]$ compression direction and therefore possess a high Schmid factor.
\end{abstract}

DOI: 10.1103/PhysRevB.64.014202

PACS number(s): 61.72.Hh, 61.72.Ff, 62.20.Fe

\section{INTRODUCTION}

An icosahedral phase in the zinc-based alloy Zn-Mg-RE, where RE stands for a rare-earth element, was first observed by Luo et al. ${ }^{1}$ A highly ordered face-centered icosahedral phase $^{2,3}$ coexisting with a simple-cubic icosahedral phase ${ }^{4}$ in $\mathrm{Zn}-\mathrm{Mg}-\mathrm{RE}$ ( $\mathrm{RE}=\mathrm{Y}, \mathrm{Tb}, \mathrm{Dy}, \mathrm{Er}, \mathrm{Ho})$ was later reported. The formation of the simple-cubic and face-centered phase was shown to be dependent on the growth conditions ${ }^{4}$ and on the content of rare-earth elements. ${ }^{5}$ Based on the investigation of the primary solidification area of the icosahedral phase in the $\mathrm{Zn}-\mathrm{Mg}-\mathrm{Y}$ alloy phase diagram, ${ }^{6}$ large single quasicrystals of face-centered icosahedral Zn-Mg-Dy were grown using the self-flux technique. ${ }^{7,8}$

The plastic macroscopic deformation properties of icosahedral $\mathrm{Zn}-\mathrm{Mg}$-Dy single quasicrystals were first studied by Heggen et al. ${ }^{8}$ In comparison to other quasicrystals such as icosahedral Al-Pd-Mn, ${ }^{9}$ the stress-strain curves of $\mathrm{Zn}-\mathrm{Mg}$-Dy show some unusual features. An extraordinary strong yield drop of about $40 \%$ of the upper yield stress and a subsequent constant flow-stress level up to high strains was found. Microstructural investigations on plastically deformed $\mathrm{Zn}$ Mg-Dy single quasicrystals demonstrated that plastic deformation is mediated by a dislocation mechanism. ${ }^{10}$ Furthermore, the presence of planar defects in deformed and heat-treated materials was observed.

In this paper, we present a detailed study of defect structures in icosahedral Zn-Mg-Dy quasicrystals. We give a comparative characterization of dislocations and planar defects in deformed, as-cast and heat-treated material.

\section{EXPERIMENT}

Icosahedral $\mathrm{Zn}-\mathrm{Mg}$-Dy single quasicrystals with diameters of up to $15 \mathrm{~mm}$ were grown using the self-flux technique. ${ }^{11,7}$ The starting materials were sealed in a molybdenum crucible under vacuum conditions with the composition 46 at. $\% \mathrm{Zn}, 51$ at. $\% \mathrm{Mg}$, and 3 at. \% Dy. The crucible was placed into a chamber furnace and subjected to heat treatment according to the following scheme: Heating to $700^{\circ} \mathrm{C}$, holding at this temperature for $7 \mathrm{~h}$, homogenizing the melt; slow cooling from $650^{\circ} \mathrm{C}$ to $575^{\circ} \mathrm{C}$ at $1{ }^{\circ} \mathrm{h}^{-1}$ and from $575^{\circ} \mathrm{C}$ to $480^{\circ} \mathrm{C}$ at $0.6^{\circ} \mathrm{h}^{-1}$. At $480{ }^{\circ} \mathrm{C}$ the excess liquid was decanted.

The material grown was characterized metallographically and by transmission electron microscopy. Optical- and scanning electron microscopy revealed the absence of second phases. The composition of the quasicrystal was determined as 62.6 at. $\% \mathrm{Zn}, 28.3$ at. \% $\mathrm{Mg}$, and 9.1 at. \% Dy by means of inductively coupled plasma optical emission spectroscopy. The electron diffraction patterns obtained in the transmission electron microscope (TEM) show sharp spots properly aligned along the systematic rows, indicating a high structural perfection of the quasicrystals. The presence of the face-centered icosahedral phase was verified by analyzing the electron diffraction patterns along twofold axes of the quasicrystal structure, which show fundamental as well as superlattice reflections (cf. Rodewald et al. ${ }^{5}$ ).

For plastic deformation cuboid-shaped samples of about $3.2 \times 1.3 \times 1.3 \mathrm{~mm}^{3}$ in size were cut from the single quasicrystals. The long axis, i.e., the compression direction was aligned parallel to a twofold direction. We performed uniaxial deformation experiments in compression at strain rates of $10^{-5}$ and $10^{-6} \mathrm{~s}^{-1}$ in a temperature range between $490{ }^{\circ} \mathrm{C}$ and $530{ }^{\circ} \mathrm{C}$ corresponding to $87.4 \%$ and $92.0 \%$ of the absolute melting temperature, respectively. The results of the macroscopic deformation experiments have been described in detail by Heggen et al. ${ }^{8}$

The microstructural investigations were performed on material subjected to different treatments. Four types of samples were investigated: $(A)$ as-grown material, $(B)$ heat-treated material annealed at temperatures equal to the deformation temperatures 500 and $510^{\circ} \mathrm{C}$ and subsequently quenched in water, $(C)$ material deformed up to a plastic strain of $0.2 \%$ corresponding to the upper yield point, $(D)$ material deformed up to a plastic strain of $1.6 \%$ corresponding to the 


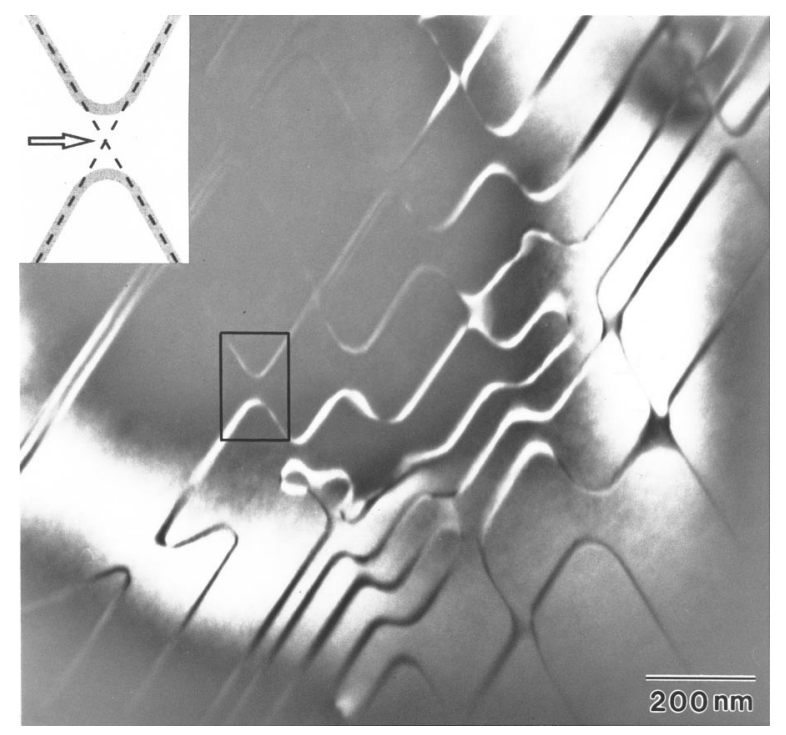

FIG. 1. Bright-field image of planar defects in icosahedral Zn$\mathrm{Mg}$-Dy single quasicrystals. The inset shows a schematic of the boxed region.

steady-state regime in the stress-strain curve. After plastic deformation, type $C$ and $D$ materials were rapidly unloaded and quenched in water to preserve the structural state during the deformation experiment as closely as possible. Specimens for transmission electron microscopy were produced by standard techniques, i.e., subsequent slicing, grinding, polishing, and argon-ion milling.

The microstructural investigations were carried out in JEOL 4000FX and 2000EX TEMs operated at $200 \mathrm{kV}$. The dislocation density was determined from about ten brightfield micrographs taken at different positions of every specimen by counting the intersections of dislocations with the specimen surfaces. The dislocation density was then calculated according to

$$
\rho=\frac{N}{A},
$$

where $N$ is the number of intersections of dislocation lines with both surfaces of the specimen and $A$ is the area of the sample region on the micrograph. ${ }^{12}$

\section{RESULTS}

In deformed samples $C$ and $D$ and in heat-treated samples $B$ microstructural analysis reveals the presence of defects showing a bandlike image contrast. A bright-field Braggcontrast image of such defects is shown in Fig. 1. The inset shows a schematic of the boxed region. Specimen-tilting experiments in the TEM, leading to changing widths of the defect contrast, clearly show that the defects are planar. The figure shows long and straight segments of planar defects connected by regions of strong curvature. Many not directly connected straight segments are, however, found to be lined up perfectly, indicating that these segments are apparently localized in the same plane (dashed lines in inset). At the crossings of such planes, i.e., between face-to-face oriented strongly curved regions (arrow in inset), the same contrast as

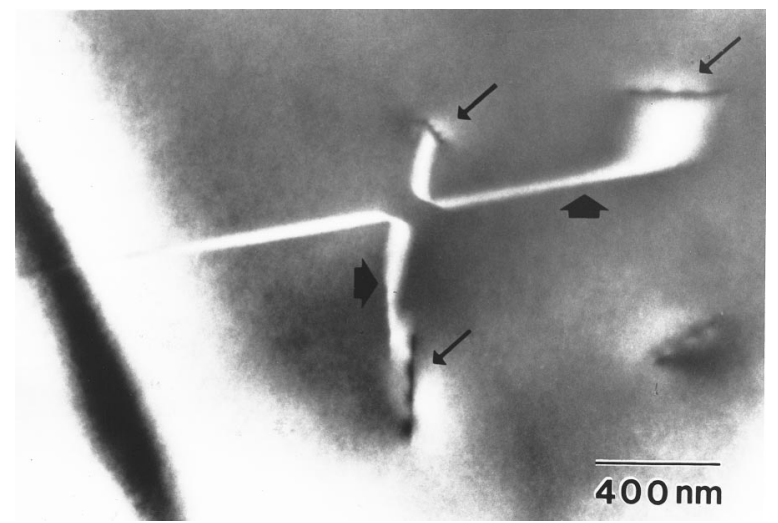

FIG. 2. Bright-field image of a heat-treated sample $B$. Dislocations are marked by thin arrows, planar defects in different orientations are marked by thick arrows.

in the image background is observed.

Figure 2 shows a Bragg-contrast image of a heat-treated sample $B$. The planar defects can be recognized as bands of uniform bright contrast against the darker background. Parts of the planar-defect contrast, showing different widths resulting from different plane orientations, are marked by thick arrows. The planar defects are terminated by dislocations (thin arrows), showing a dark line contrast. In the central part of the figure, we find a formation similar to that shown in the inset of Fig. 1. At the crossing of the planes containing the vertical and the almost horizontally seen defect contrast, the same gray contrast as the background is observed. The horizontal and vertical straight segments are connected by strongly curved segments.

Figure 3 shows a series of dark-field Bragg-contrast images obtained close to a twofold [0/0 0/0 0/2] zone axis. In the bottom right part of the figure, the corresponding diffraction pattern is seen. The reflections of a fivefold-systematic row $\left(\mathbf{g}_{51}\right.$ to $\left.\mathbf{g}_{55}\right)$ used for imaging under two-beam conditions are marked with arrowheads. The indexing of the corresponding diffraction vectors according to the system of Cahn et al. ${ }^{13}$ is given in Table I. If the superstructure reflections $\mathbf{g}_{51}, \mathbf{g}_{52}$, and $\mathbf{g}_{54}$ are used for imaging under two-beam conditions, the planar defects are in contrast, whereas if the fundamental reflections $\mathbf{g}_{53}$ and $\mathbf{g}_{55}$ are used they are out of contrast and only a weak residual contrast can be observed. The same conditions are found using other reflections for imaging, e.g., those in threefold or other fivefold systematic rows. Generally, we find that if superstructure reflections are

TABLE I. Indices of the $g$-vectors used for imaging in Fig. 3. The spots are subdivided into fundamental spots $(f)$ and superstructure spots $(s)$.

\begin{tabular}{lccc}
\hline \hline & $h / h^{\prime}, k / k^{\prime}, l / l^{\prime}$ & Type & Planar defects visible \\
\hline $\mathbf{g}_{51}$ & $(0 / 1,1 / 1,0 / 0)$ & $s$ & yes \\
$\mathbf{g}_{52}$ & $(1 / 1,1 / 2,0 / 0)$ & $s$ & yes \\
$\mathbf{g}_{53}$ & $(1 / 2,2 / 3,0 / 0)$ & $f$ & no \\
$\mathbf{g}_{54}$ & $(2 / 3,3 / 5,0 / 0)$ & $s$ & yes \\
$\mathbf{g}_{55}$ & $(2 / 4,4 / 6,0 / 0)$ & $f$ & no \\
\hline \hline
\end{tabular}



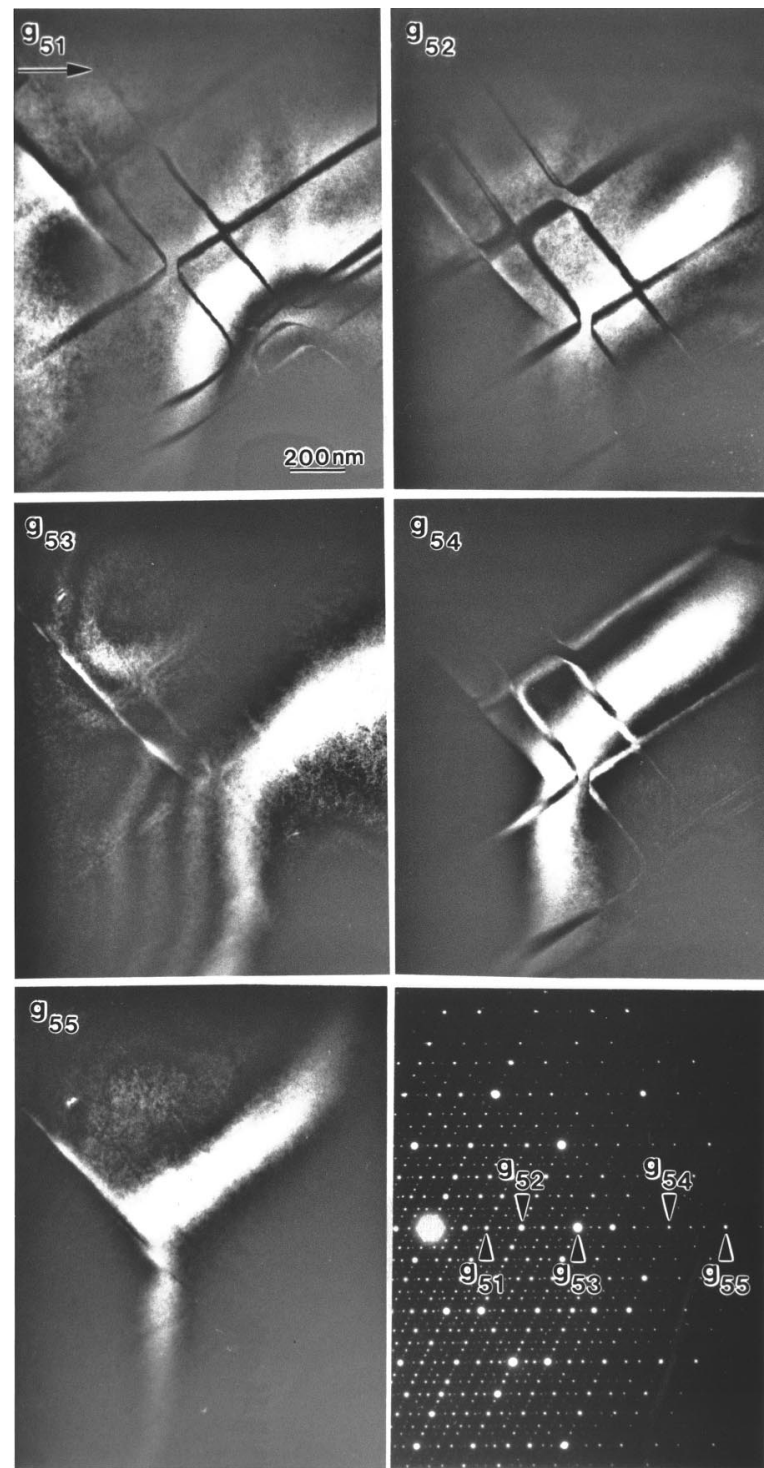

FIG. 3. Centered dark-field images, using diffraction vectors of a fivefold systematic row. In the bottom right section the twofold diffraction pattern is shown, with arrowheads marking the corresponding reflections (indices in Table I).

used for imaging, the planar defects are in contrast, whereas if fundamental spots are used, the planar defects are out of contrast.

The density of planar defects depends on the treatment of

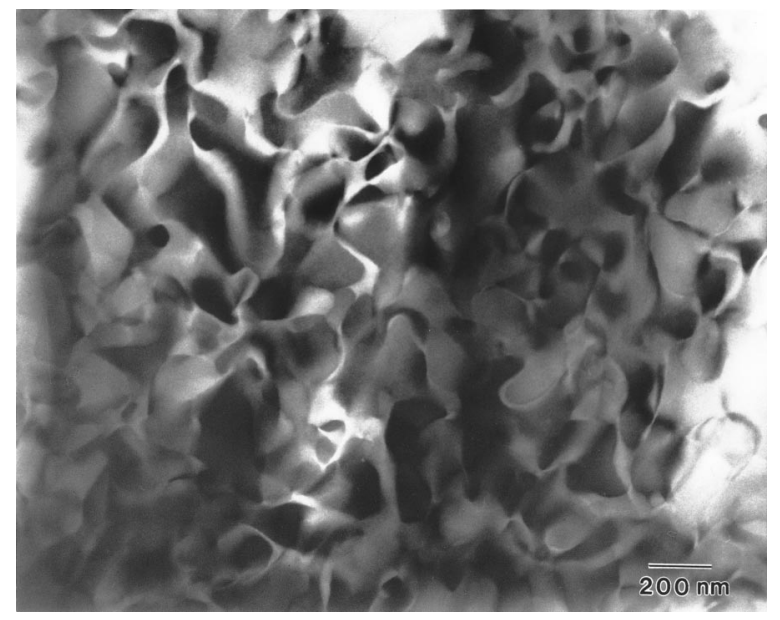

FIG. 4. Bright-field image of sample $D$ deformed at $500{ }^{\circ} \mathrm{C}$ up to $1.6 \%$ of plastic strain. A high density of planar defects is visible.

the sample. While in samples $A$ no planar defects could be detected, we find that in the heat-treated sample $B 20 \%$ of the dislocations present in the material are connected to planar defects. Their density further increases with increasing plastic strain. In sample $C$, which was deformed up to the upper yield point, $52 \%$ of the dislocations are connected to planar defects. In sample $D$, deformed up to the steady-state regime, the planar-defect density is extremely high. Since the number of defect-plane crossings is accordingly large, all segments show a strong curvature. This leads to a rather complex defect structure as shown in Fig. 4. Here, the density of planar defects is too high to assign dislocations to the planar defects.

The dislocation densities in the as-grown $(A)$ and in the heat-treated $(B)$ sample were determined as $6 \times 10^{6}$ and 5 $\times 10^{7} \mathrm{~cm}^{-2}$, respectively. In the deformed samples we find distinctly higher dislocation densities of $2.2 \times 10^{8}$ and 5.6 $\times 10^{9} \mathrm{~cm}^{-2}$ at strains of $0.2(C)$ and $1.6 \%(D)$, respectively. These results are compiled in Table II.

Plane normals of planar defects were determined by specimen-tilting experiments in the TEM. In a sample of type $B$, which was heated at $510^{\circ} \mathrm{C}$ for $7 \mathrm{~h}$ and subsequently quenched in water, the plane normals for 58 examples of planar defects could be determined. In these samples, the planar defects are often straight over distances of several microns and are frequently arranged in parallel (Fig. 5). We found that $90 \%$ of the plane normals were oriented perpen-

TABLE II. Dislocation density and appearance of planar defects in samples of the four categories.

\begin{tabular}{lcl}
\hline \hline Sample & $\begin{array}{c}\text { Dislocation density } \\
\rho / \mathrm{cm}^{-2}\end{array}$ & \multicolumn{1}{c}{ Planar defects } \\
\hline$(A)$ as-grown & $6 \times 10^{6}$ & $\begin{array}{l}\text { No planar defects found } \\
20 \% \text { of dislocations attached to } \\
\text { planar defects } \\
52 \% \text { of dislocations attached to } \\
\text { planar defects }\end{array}$ \\
$\begin{array}{l}\text { (C) Annealed for } 500{ }^{\circ} \mathrm{C} \text { deformed to the } 500{ }^{\circ} \mathrm{C} \\
\text { upper yield point }\left(\varepsilon_{\text {plast }}=0.2 \%\right)\end{array}$ & $2.4 \times 10^{8}$ & $\begin{array}{l}\text { Extremely high density of planar } \\
\text { defects (Fig. 4) }\end{array}$ \\
$\begin{array}{l}\text { (D) At } 500{ }^{\circ} \mathrm{C} \text { deformed to the } \\
\text { steady-state regime }\left(\varepsilon_{\text {plast }}=1.6 \%\right)\end{array}$ & $5.6 \times 10^{9}$ & \\
\hline \hline
\end{tabular}




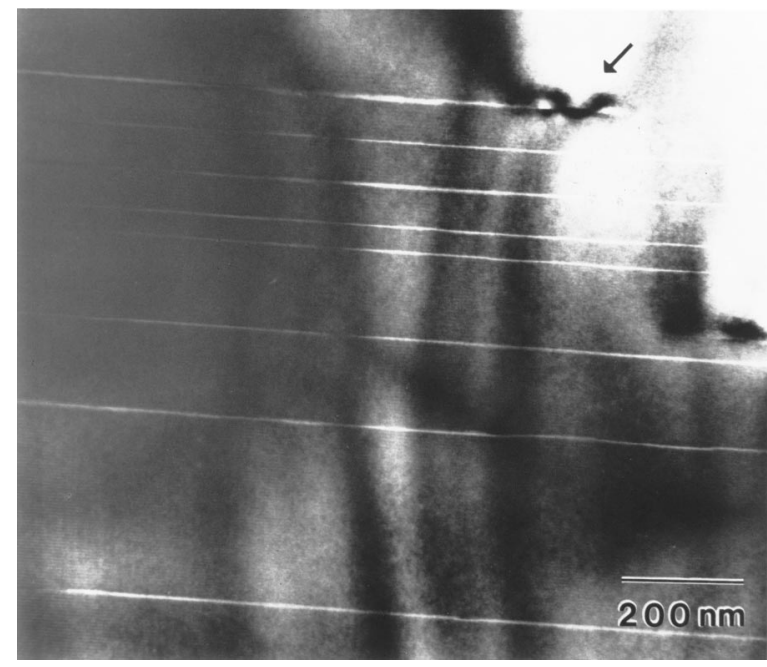

FIG. 5. Bright-field image of a sample $B$ with a comparatively high density of planar defects. The planar defects are arranged in parallel on fivefold planes, viewed in an edge-on orientation. A dislocation terminating a planar defect is marked by a thin arrow.

dicular to fivefold directions and $10 \%$ were oriented perpendicular to threefold directions. The density of planar defects is found to be rather inhomogeneous. Besides regions of high planar-defect density (Fig. 5), other regions with considerably lower densities can be found.

In deformed samples of type $C$ at a specimen orientation in the TEM with the electron beam parallel to the $[0 / 00 / 0$ $0 / 2$ ] compression direction most planar-defect normals are inclined with respect to the viewing direction. Only very few examples of planar defects in edge-on orientation can be found. In particular, we most frequently find planar defects with normals oriented parallel to pseudotwofold $[1 / 1,2 / 1$, 1/2] directions. These make an angle of $46^{\circ}$ with the compression direction. Less frequently, planar-defect normals parallel to pseudotwofold $\left[\begin{array}{lll}1 / 2 & 1 / 1 & 2 / 1\end{array}\right]$ directions $\left(54^{\circ}\right.$ inclination to compression axis) and fivefold [0/0 $1 / 0$ 0/1] and [0/1 $0 / 01 / 0]$ directions $\left(32^{\circ}\right.$ and $58^{\circ}$ inclination to the compression axis, respectively) are observed. In some cases the plane normals could not be assigned to a low-index direction of the quasilattice, but their normals were found to make an angle close to $45^{\circ}$ with the compression direction. In samples $D$, which were deformed up to higher strains, the orientations of the planar defects could not be determined, due to their high density and the strong curvature of the segments (Fig. 4). Planar segments, straight over some $100 \mathrm{~nm}$ as in samples $B$ or $C$, could not be observed.

For some examples we determined Burgers vector directions of dislocations terminating planar defects by means of contrast-extinction experiments. In a sample of type $B$ we found a planar defect having a normal parallel to the fivefold $[-1 / 0,0 / 1,0 / 0]$ direction to be terminated by dislocation with a twofold $[-1 / 0,1 / 1,0 / 1]$ physical-space Burgersvector direction. For a planar defect with a threefold [1/2, $0 / 1,0 / 0]$ plane normal, we found a twofold $[1 / 1,0 / 1,1 / 0]$ Burgers-vector direction in physical space for the dislocation attached. The angles between the physical-space components of the Burgers vector and the normals of the planar defects are thus $32^{\circ}$ and $21^{\circ}$, respectively. In a deformed sample $C$, dislocations attached to planar defects with pseudotwofold $[1 / 1,2 / 1,1 / 2]$ normals were investigated. A twofold $[-1 /$ $-1,0 /-1,1 / 0]$ physical-space direction of the Burgers vector was found in three cases and a $[-1 / 0,-1 /-1,0 / 1]$ twofold Burgers vector was found in two cases. The angles between the parallel components of the Burgers vectors and plane normals thus amount to $65^{\circ}$ and $75^{\circ}$, respectively.

\section{DISCUSSION}

In this paper, we present the contrast-extinction study of planar defects in icosahedral Zn-Mg-Dy quasicrystals. It was shown that these defects are in contrast if superstructure reflections of the face-centered hyperlattice are used for imaging under two-beam Bragg-contrast conditions, and that their contrast is extinct if fundamental reflections are used. This gives clear evidence that these planar defects are antiphase boundaries of the face-centered ordered structure.

Antiphase boundaries (APB's) in icosahedral quasicrystals were first observed by Devaud-Rzepski et al. ${ }^{14}$ in icosahedral Al-Cu-Fe. This phase also possesses a face-centered hyperlattice. ${ }^{15}$ In their paper, Devaud-Rzepski et al. ${ }^{14}$ report on the formation of APB's in rapidly solidified icosahedral $\mathrm{Al}-\mathrm{Cu}-\mathrm{Fe}$ quasicrystals, whereas the slowly cooled alloy was reported to possess a single-domain structure. This corresponds to the results of the present work, where APB's were found in the heat-treated and quenched samples of type $B$ but not in the slowly cooled samples $A$.

The increasing density of dislocations as a result of plastic deformation provides clear evidence that plastic deformation in icosahedral $\mathrm{Zn}-\mathrm{Mg}$-Dy single quasicrystals is mediated by a dislocation mechanism. The density of APB's is also observed to increase strongly with increasing plastic strain. Furthermore, we find that higher percentages of dislocations are attached to APB's at higher dislocation densities. These facts strongly indicate that the APB's are introduced into the structure by moving dislocations. In terms of the sixdimensional (6D) hyperlattice, this observation can be understood as a result of the movement of hyperlattice partials, the Burgers vectors of which do not correspond to a translation vector of the $6 \mathrm{D}$ lattice. In the highly ordered face-centered hyperlattice, dislocations with Burgers vectors corresponding to translation vectors of the $6 \mathrm{D}$ lattice would be hyperlattice superdislocations, the projection of which into physical space leads to energetically unfavorably large Burgers-vector magnitudes. A splitting into hyperlattice partials, a mechanism to be seen in analogy to superpartial splitting in ordered fcc alloys, leads to dislocations with energetically more favorable shorter Burgers vectors, the movement of which, however, causes the formation of APB's.

For some examples in undeformed and deformed samples $B$ and $C$ it was possible to simultaneously determine the directions of dislocation Burgers vectors and the normals of their associated APB's. The results show that the Burgers vectors are not parallel to the ABP's, that is, to their planes of motion. This shows that the movement of the dislocations is a mixed process of glide and climb. In the heat-treated material $B$ angles of $21^{\circ}$ and $32^{\circ}$ between the Burgers vectors and the APB normals are observed, whereas in the de- 


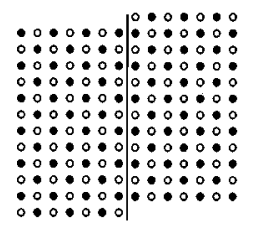

a

FIG. 6. Schematic of a two-dimensional ordered lattice, including a vertically aligned APB (a). After introducing an additional horizontal APB (b), the lattice is separated into two types of domains (denoted as $D_{1}$ and $D_{2}$ ). Movement of the APB's in the direction of the arrows (c) leads to a reduction of the total energy.

formed material $C$ we find larger angles of $65^{\circ}$ and $75^{\circ}$. Thus, in the first case the climb component and in the second case the glide component is dominant. This observation indicates that the glide-climb proportion increases as a result of plastic deformation. This result corresponds to those of earlier studies by Wang et al. ${ }^{16}$ and Caillard et al. ${ }^{17}$ in which dislocation-induced planar faults and their strain-field direction or the Burgers-vector direction of their bounding dislocations were analyzed by TEM. In both studies, directions parallel to the fault-plane normals were found. Caillard et $a l .{ }^{17}$ interpreted these observations in terms of pure climb.

In heat-treated and quenched samples of type $B$, the APB's are in fivefold and threefold planes of the quasilattice. This indicates that without the presence of a tended external force the movement of partial dislocations preferentially takes place in low-indexed planes. A different situation is encountered in the deformed samples $C$, where a directional external stress field governs the force on the dislocations. Here APB's in pseudotwofold $\{1 / 1,2 / 1,1 / 2\}$ planes are found to be dominant, which are tilted by $46^{\circ}$ with respect to the compression direction and therefore resolve a high shear stress. The other, less frequently observed planes also possess rather favorable inclinations to the compression direction. Moreover, APB's in planes, not perpendicular to lowindex directions but rather close to $45^{\circ}$ orientations with respect to the compression direction have been observed. Thus we can conclude that in the presence of a tended external force, the maximization of the resolved shear stress, rather than the retention of a low-index plane is the deciding parameter for the choice of the plane of dislocation motion. This result is supported by the observation that the movement of dislocations does not take place by pure glide but rather involves a climb component. Due to the presence of this additional mode of movement, the motion of dislocations is less bound to low-index planes. A dislocation is able to accommodate its motion such that the overall plane of movement can most effectively contribute to relieve the tended applied stress.

In an ordered face-centered icosahedral structure, there are only two types of domains and one type of $\mathrm{APB}^{15}$ and therefore stable networks of APB's do not exist. It can be shown that crossings of APB's lead to their annihilation at the line of intersection. This is illustrated schematically in Fig. 6 for a two-dimensional ordered structure. The APB created by the movement of a partial dislocation [Fig. 6(a)] is crossed by a second APB [Fig. 6(b)]. At the crossing point, the original structure is recovered, leading to the presence of

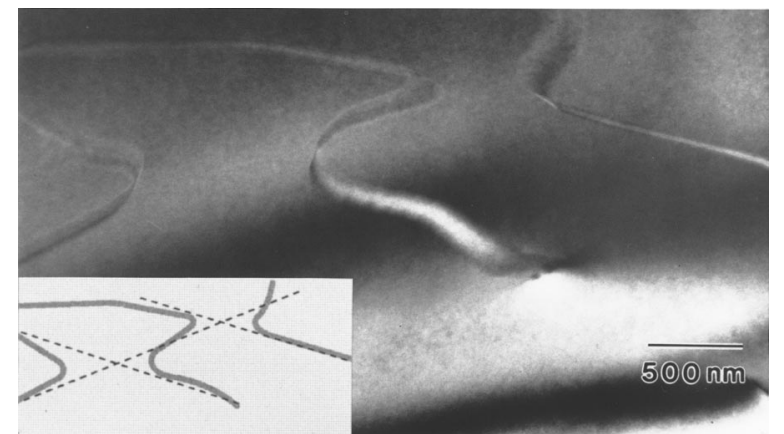

FIG. 7. Bright-field image of a heat-treated and slowly cooled sample. The planar faults are less straight due to longer subjection to higher temperatures. The dashed lines in the inset show the planes of motion of the dislocations.

three domains of the two types, $D_{1}$ and $D_{2}$. In order to decrease the total energy, the fault area is reduced by diffusive rearrangement of atoms, leading to a situation as shown in Fig. 6(c). The further increase of the radius of the curved segments, i.e., further movement of the APB's in the direction of the arrows in Fig. 6(c) leads to a further reduction of the total energy. Therefore, the arrangement of APB's is not stable. A preceding increase of the radius of the curved segments, however, involves the rearrangement of increasing numbers of atoms, and therefore becomes slower and slower. The experimentally observed arrangements of APB's at the crossings of planes of dislocation motion as shown in Figs. 1 and 2 corresponds to the situation shown in Fig. 6(c). This scenario of the formation of the APB-crossing arrangement observed is supported by the additional study of a sample heated for $2 \mathrm{~h}$ at $500{ }^{\circ} \mathrm{C}$ which, in contrast to samples of type $B$, was not rapidly quenched but slowly cooled at a rate of $300{ }^{\circ} \mathrm{Ch}^{-1}$. The microstructural investigation of these samples (Fig. 7) shows that the shape of the APB's is less straight compared with those in quenched samples (Figs. 1 and 2). Figure 7 shows two crossings of APB's (see schematic in inset). In contrast to the rapidly-quenched material $B$, the distance from the APB's to the intersecting lines of the glide planes is much higher. Due to the slower cooling rate, the material is subjected to high temperatures for a longer time. This leads to a further progression of the APB arrangement towards a state of lower total energy.

In conclusion, we have presented a detailed microstructural investigation of defects in icosahedral $\mathrm{Zn}-\mathrm{Mg}$-Dy single quasicrystals. We have shown that in this material the movement of partial dislocations leads to the introduction of hyperlattice APB's, and we have explained the observed arrangements of APB's in terms of diffusional reordering of atoms. Since the APB's allow an unambiguous determination of the plane of motion of the dislocations, a correlation between this plane and the dislocations Burgers vector was possible. This indicates that dislocation motion in this material occurs at high temperatures by both glide and climb.

\section{ACKNOWLEDGMENTS}

We are grateful to I. R. Fisher and P. C. Canfield for support in the development of the quasicrystal-growth procedures and to C. Thomas and M. Beyss for performing the growth. 
${ }^{1}$ Z. Luo, S. Zhang, Y. Tang, and D. Zhao, Scr. Metall. Mater. 28, 1513 (1993).

${ }^{2}$ A. Niikura, A. P. Tsai, A. Inue, and T. Masumoto, Philos. Mag. Lett. 69, 351 (1994).

${ }^{3}$ A. P. Tsai, A. Niikura, A. Inue, and T. Masumoto, Philos. Mag. Lett. 70, 169 (1994).

${ }^{4}$ A. Niikura, A. P. Tsai, A. Inue, and T. Masumoto, Jpn. J. Appl. Phys., Part 1 33, 1538 (1994).

${ }^{5}$ M. Rodewald, A. Langsdorf, W. Assmus, and H. Fuess, in Proceedings of the 6th International Conference on Quasicrystals, Tokyo 1998, edited by S. Takeuchi and T. Fujiwara (World Scientific, Singapore, 1997), p. 51.

${ }^{6}$ A. Langsdorf, F. Ritter, and W. Assmus, Philos. Mag. Lett. 75, 381 (1997).

${ }^{7}$ I. R. Fisher, Z. Islam, A. F. Panchula, K. O. Cheon, M. J. Kramer, P. C. Canfield, and A. I. Goldman, Philos. Mag. B 77, 1601 (1998).

${ }^{8}$ M. Heggen, M. Feuerbacher, P. Schall, H. Klein, I. R. Fisher, P.
C. Canfield, and K. Urban, Philos. Mag. Lett. 80, 129 (2000).

${ }^{9}$ M. Feuerbacher, C. Metzmacher, M. Wollgarten, K. Urban, B. Baufeld, M. Bartsch, and U. Messerschmidt, Mater. Sci. Eng., A 226-228, 943 (1997).

${ }^{10}$ M. Heggen, M. Feuerbacher, P. Schall, H. Klein, I. R. Fisher, P. C. Canfield, and K. Urban, Mater. Sci. Eng., A 294-296, 781 (2000).

${ }^{11}$ P. C. Canfield and Z. Fisk, Philos. Mag. B 65, 1117 (1992).

${ }^{12}$ R. K. Ham and N. G. Sharpe, Philos. Mag. 6, 1193 (1961).

${ }^{13}$ J. W. Cahn, D. Shechtman, and D. Gratias, J. Mater. Res. 1, 13 (1986).

${ }^{14}$ J. Devaud-Rzepski, A. Quivy, Y. Calvayrac, M. CornierQuiquandon, and D. Gratias, Philos. Mag. B 60, 855 (1989).

${ }^{15}$ S. Ebalard and F. Spaepen, J. Mater. Res. 5, 62 (1989).

${ }^{16}$ R. Wang, M. Feuerbacher, W. Yang, and K. Urban, Philos. Mag. A 78, 273 (1998).

${ }^{17}$ D. Caillard, G. Vanderschaeve, L. Bresson, and D. Gratias, Philos. Mag. A 80, 237 (2000). 